\title{
THE CUP PRODUCT OF BROOKS QUASIMORPHISMS
}

\author{
MICHELLE BUCHER AND NICOLAS MONOD
}

\begin{abstract}
We prove the vanishing of the cup product of the bounded cohomology classes associated to any two Brooks quasimorphisms on the free group. This is a consequence of the vanishing of the square of a universal class for tree automorphism groups.
\end{abstract}

\section{INTRODUCTION}

Although bounded cohomology found a great variety of applications, it remains so mysterious that even for a (non-abelian) free group $F$ of finite rank, we do not know much about it.

More precisely, beyond the trivial case of $\mathrm{H}_{\mathfrak{b}}^{1}(F, \mathbf{R})=0$, it is known that both $\mathrm{H}_{\mathfrak{b}}^{2}(F, \mathbf{R})$ and $\mathrm{H}_{\mathrm{b}}^{3}(F, \mathbf{R})$ are infinite-dimensional. However, $\mathrm{H}_{\mathrm{b}}^{n \geq 4}(F, \mathbf{R})$ remains completely unknown; in particular, we do not know whether $\mathrm{H}_{\mathrm{b}}^{4}(F, \mathbf{R})$ vanishes or not.

The first infinite family of non-trivial classes in $\mathrm{H}_{b}^{2}(F, \mathbf{R})$ are provided by Brooks quasimorphisms [1] (anticipated by Johnson [7, 2.8] and Rhemtulla [12]); we recall their definition. Pick any reduced word $w$ in a choice of free generators for $F$ and consider the counting function $f_{w}: F \rightarrow \mathbf{R}$ defined on $g \in F$ by

$$
f_{w}(g)=\sharp\{\text { occurrences of } w \text { in } g\}-\sharp\left\{\text { occurrences of } w \text { in } g^{-1}\right\} .
$$

If $w$ is reduced to one letter (or trivial), then $f_{w}$ is a homomorphism. In all other cases, $f_{w}$ is a quasimorphism and defines a non-trivial class $\beta_{w} \in \mathrm{H}_{\mathrm{b}}^{2}(F, \mathbf{R})$ unless $w$ is conjugated to a power of a letter. The space spanned by all these $\beta_{w}$ is infinite-dimensional [1] [8] and is dense in $\mathrm{H}_{b}^{2}(F, \mathbf{R})$ for a suitable topology of pointwise convergence [4, 5.7]. (Following Brooks, we allow overlaps when counting occurrences, whilst other authors do not; see [5. p. 251] for the density in our setting.)

The aim of this note is to show that the cup product of any two elements in this dense sub-space vanishes in $\mathrm{H}_{\mathrm{b}}^{4}(F, \mathbf{R})$.

Theorem 1. Let $\beta_{w}, \beta_{w^{\prime}} \in \mathrm{H}_{\mathrm{b}}^{2}(F, \mathbf{R})$ be the bounded cohomology classes associated to two Brooks quasimorphisms on $F$.

Then $\beta_{w} \smile \beta_{w^{\prime}}=0$ in $\mathrm{H}_{\mathrm{b}}^{4}(F, \mathbf{R})$.

We were informed by N. Heuer that he independently obtained a similar result [6] by methods completely different from ours.

We can give a rather transparent proof of Theorem 1 by realizing bounded cohomology with the aligned chains that we introduced in [2]. This simplifies the combinatorics and allows us to exhibit a natural explicit coboundary for the cup product.

Moreover, we can carry out this task at once for all $w, w^{\prime}$ simultaneously - by working instead with the universal class $[\omega]$ that we now proceed to define (similar constructions were considered in [10, §2], in [11, 7.11] and in [3, §9]). 
Let $T=(V, E)$ be a locally finite tree with Serre's conventions, which means in particular that an element of $E$ represents an oriented edge and that $E$ is endowed with a fixed-pointfree involution $e \mapsto \bar{e}$ reversing the orientation. We denote by $P$ the set of paths, namely sequences $p=\left(e_{1}, \ldots, e_{n}\right)$ of successive edges $e_{i} \in E$ without backtracking. The reverse path is $\bar{p}=\left(\bar{e}_{n}, \ldots, \bar{e}_{1}\right)$ and $n$ is the length of $p$. Given two vertices $x, y$ we denote by $[x, y]$ the path connecting them. The path module $\mathbf{R}_{\text {alt }}[P]$ is the Aut $(T)$-module of all elements of the free vector space $\mathbf{R}[P]$ that change sign when replacing a path by its reverse. We define an $\operatorname{Aut}(T)$-equivariant map $\vartheta: V^{2} \rightarrow \mathbf{R}_{\text {alt }}[P]$ by setting

$$
\vartheta\left(x_{0}, x_{1}\right)(p)= \pm 1
$$

if $p$ (respectively $\bar{p}$ ) is contained as a sub-path in $\left[x_{0}, x_{1}\right]$, and 0 in all other cases. We define

$$
\omega=d \vartheta: V^{3} \rightarrow \mathbf{R}_{\text {alt }}[P]
$$

as the coboundary of $\vartheta$. We recall here that $d$ will always be the usual alternating sum of the maps omitting the individual variables; we refer to the preliminaries below for explicit values of $\omega$.

In order to view $\omega$ as a cocycle in bounded cohomology, we need to specify a norm on $\mathbf{R}_{\text {alt }}[P]$; of course, $\vartheta$ should be unbounded for this norm since otherwise the class of $\omega$ would be trivial. The specific norm is however not too relevant; one property we want is that, when restricted to the free vector space on the set of paths of length $n$, it is equivalent to the $\ell^{1}$ norm $\|\cdot\|_{n, 1}$. One explicit choice is the norm $\|\cdot\|_{\text {path }}=\sum_{n \geq 1} \frac{1}{n !}\|\cdot\|_{n, 1}$ whose normalisation factor $1 / n$ ! is an arbitrary way to ensure uniform boundedness statements in the proofs.

Furthermore, we write $\mathscr{P}$ for the completion of $\mathbf{R}_{\text {alt }}[P]$. Indeed, even though our arguments will be explicit and finitary, the general tools of continuous bounded cohomology work best with Banach spaces.

A choice of free generators for the free group $F$ determines an embedding of $F$ into the automorphism group of the corresponding tree $T$. We view $\omega$ as a cocycle for the continuous bounded cohomology $\mathrm{H}_{\mathrm{cb}}^{*}$ of the locally compact group Aut $(T)$.

Moreover, every path in $T$ is labelled by a reduced word in $F$. Thus, given a reduced word $w$, we can define an $F$-invariant bounded linear form $\lambda_{w}$ on $\mathbf{R}_{\text {alt }}[P]$, hence also on $\mathscr{P}$, by specifying its values on individual paths as follows:

$$
\lambda_{w}(p)=\left\{\begin{aligned}
1 & \text { if } w \text { labels } p \\
-1 & \text { if } w \text { labels } \bar{p} \\
0 & \text { otherwise. }
\end{aligned}\right.
$$

This definition ensures that if $g \in F$ labels $\left[x_{0}, x_{1}\right]$, then

$$
\lambda_{w} \circ \vartheta\left(x_{0}, x_{1}\right)=f_{w}(g) \text {. }
$$

Therefore, we deduce immediately the following relation between the universal class $[\omega]$ and individual quasimorphisms.

Proposition 2. Let $\beta_{w} \in \mathrm{H}_{\mathfrak{b}}^{2}(F, \mathbf{R})$ be the bounded cohomology class associated to a Brooks quasimorphism on $F$ for the chosen generators. Then $\beta_{w}$ is the image of the class of $\omega$ under the map

$$
\mathrm{H}_{\mathrm{cb}}^{2}(\operatorname{Aut}(T), \mathscr{P}) \stackrel{\text { rest }}{\longrightarrow} \mathrm{H}_{\mathrm{b}}^{2}(F, \mathscr{P}) \stackrel{\left(\lambda_{w}\right)_{*}}{\longrightarrow} \mathrm{H}_{\mathrm{b}}^{2}(F, \mathbf{R}),
$$

where the first arrow is the restriction map and the second is induced by $\lambda_{w}$. 
The cup product of two elements of $\mathrm{H}_{\mathrm{cb}}^{2}(\operatorname{Aut}(T), \mathscr{P})$ is a class in $\mathrm{H}_{\mathrm{cb}}^{4}$ with values in the tensor product module $\mathscr{P} \otimes \mathscr{P}$, which we can also (projectively) complete to $\mathscr{P} \widehat{\otimes} \mathscr{P}$ (see the preliminaries for the norm). The naturality of the cup product now implies:

Corollary 3. Given two reduced words $w$ and $w^{\prime}$, we keep all the above notation.

Then $[\omega] \smile[\omega]$, viewed as a class with coefficients in $\mathscr{P} \widehat{\otimes} \mathscr{P}$, is mapped to $\beta_{w} \smile \beta_{w^{\prime}}$

$$
\mathrm{H}_{\mathrm{cb}}^{4}(\operatorname{Aut}(T), \mathscr{P} \widehat{\otimes} \mathscr{P}) \longrightarrow \mathrm{H}_{\mathrm{b}}^{4}(F, \mathbf{R})
$$

under the restriction followed by $\left(\lambda_{w} \otimes \lambda_{w^{\prime}}\right)_{*}$.

In view of Corollary 3 , Theorem 1 is now an immediate consequence of the following vanishing result for the square of the universal class $[\omega]$.

Theorem 4. Let $\mathrm{T}$ be a locally finite tree.

Then the class of $\omega \smile \omega$ vanishes in $\mathrm{H}_{\mathrm{cb}}^{4}(\operatorname{Aut}(T), \mathscr{P} \widehat{\otimes} \mathscr{P})$.

The remainder of this note is devoted to the proof of Theorem 4

\section{PRELIMINARIES}

The cup-product of bounded cocycles ranging in $\mathbf{R}_{\text {alt }}[P]$ or $\mathscr{P}$ is trivially bounded for any cross-norm on the tensor product with underlying norm $\|\cdot\|_{\text {path }}$ on each factor. For crossnorms, we refer to [13]. We shall choose the projective cross-norm $\|\cdot\|_{\pi}$ and denote by $\mathscr{P} \widehat{\otimes} \mathscr{P}$ the corresponding completion. Since this is the largest cross-norm, the vanishing result of Theorem 4 with respect to $\|\cdot\|_{\pi}$ implies the corresponding vanishing for any other cross-norm.

We say that a path $p$ is carried by a path $q$, and write $p \sqsubset q$, if either $p$ or $\bar{p}$ is contained in $q$ as a sub-path. We attach a sign \pm 1 to these two cases, referred to as the orientation of $p$ relative to $q$. We define the interior $\operatorname{Int}(p) \subseteq V$ of a path $p$ to consist of all the vertices of the path except its two extremities.

Recall that any three vertices $x_{0}, x_{1}, x_{2} \in V$ determine a center $c \in V$ characterized as the unique common vertex of all $\left[x_{i}, x_{j}\right]$. Given a path $p$, the definition of $\omega$ now shows that $\omega(x)(p)= \pm 1$ when $p$ is carried by some $\left[x_{i}, x_{j}\right]$ and $c \in \operatorname{Int}(p)$, and that $\omega(x)(p)$ vanishes otherwise.

A path can contain at most $n-1$ sub-paths of length $n$ containing a given vertex in their interior. Therefore, considering all three configurations and two orientations, we can bound the norm of $\omega$ by

$$
\|\omega(x)\|_{\text {path }} \leq 3 \cdot 2 \cdot \sum_{n \geq 1} \frac{1}{n !}(n-1)=6,
$$

witnessing that $\omega$ is indeed uniformly bounded.

Recall that a $(q+1)$-tuple $\left(x_{0}, \ldots, x_{q}\right) \in V^{q+1}$ is aligned if the vertices $x_{0}, \ldots, x_{q}$ are contained in some geodesic segment of $T$. This tuple is furthermore said to be coherent if these $q+1$ vertices are distinct and in increasing order for one of the two linear orders induced on $\left\{x_{0}, \ldots, x_{q}\right\}$ by any such segment. We denote by $V_{\text {coh }}^{q+1} \subseteq V^{q+1}$ the set of coherent aligned tuples.

Below, we shall be particularly interested in the above description of $\omega(x)$ specialized to coherent triples $x \in V_{\text {coh. }}^{3}$. In that case, $\omega(x)(p)= \pm 1$ if $x_{1} \in \operatorname{Int}(p)$ and $p$ is carried by 
$\left[x_{0}, x_{2}\right]$, with the sign given by the orientation of $p$ relative to $\left[x_{0}, x_{2}\right]$, and vanishes in all other cases.

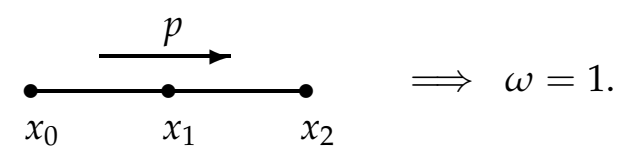

\section{THE COHERENT RESOLUTION}

Let $E$ be any isometric Banach $\operatorname{Aut}(T)$-module and recall that $\mathrm{H}_{\mathrm{cb}}^{q}(\operatorname{Aut}(T), E)$ can be computed with the (non-augmented) complex $\ell^{\infty}\left(V^{q+1}, E\right)^{\operatorname{Aut}(T)}$ of $\operatorname{Aut}(T)$-equivariant elements of the resolution

$$
0 \longrightarrow E \longrightarrow \ell^{\infty}(V, E) \longrightarrow \ell^{\infty}\left(V^{2}, E\right) \longrightarrow \ell^{\infty}\left(V^{3}, E\right) \longrightarrow \cdots
$$

(see e.g. [9, 4.5.2]). There is a natural restriction map to the complex $\ell^{\infty}\left(V_{\mathrm{coh}}^{q+1}, E\right)$ on coherent tuples, but we warn the reader that the latter is not a resolution of $E$.

Recall that an element of $\ell^{\infty}\left(V^{q+1}, E\right)$ is called alternating if any permutation $\sigma$ of the variables corresponds to the multiplication by the signature $\operatorname{sign}(\sigma)$. We denote by $\tau_{q}$ the permutation of $\{0, \ldots, q\}$ that reverses the order and observe that its signature is $(-1)^{\left\lfloor\frac{q+1}{2}\right\rfloor}$, where $\lfloor\cdot\rfloor$ denotes the integer part. Consider the $\operatorname{Aut}(T)$-equivariant involution $\hat{\tau}_{q}$ of $\ell^{\infty}\left(V_{\mathrm{coh}}^{q+1}, E\right)$ defined by $\hat{\tau}_{q}(\alpha)(x)=\operatorname{sign}\left(\tau_{q}\right) \alpha\left(x^{\tau_{q}}\right)$. Being an involution, it induces an eigenspace decomposition

$$
\ell^{\infty}\left(V_{\mathrm{coh}}^{q+1}, E\right)=\ell_{+}^{\infty}\left(V_{\mathrm{coh}}^{q+1}, E\right) \oplus \ell_{-}^{\infty}\left(V_{\mathrm{coh}}^{q+1}, E\right)
$$

which is preserved by $\operatorname{Aut}(T)$. Although $\ell^{\infty}\left(V_{\text {coh }}^{q+1}, E\right)$ is not a resolution, we have:

Proposition 5. The sub-complex

$$
0 \longrightarrow E \longrightarrow \ell_{+}^{\infty}\left(V_{\mathrm{coh}}^{1}, E\right) \longrightarrow \ell_{+}^{\infty}\left(V_{\mathrm{coh}}^{2}, E\right) \longrightarrow \ell_{+}^{\infty}\left(V_{\mathrm{coh}}^{3}, E\right) \longrightarrow \cdots
$$

is a resolution. Moreover, the map

$$
A_{q} \circ \text { rest: } \ell^{\infty}\left(V^{q+1}, E\right) \longrightarrow \ell_{+}^{\infty}\left(V_{\text {coh }}^{q+1}, E\right)
$$

from (ii) to (ii) obtained by restriction followed by the projection $A_{q}=\left(\hat{\tau}_{q}+\mathrm{Id}\right) / 2$ yields an isomorphism between $\mathrm{H}_{\mathrm{cb}}^{q}(\operatorname{Aut}(T), E)$ and the cohomology of the complex

$$
0 \longrightarrow \ell_{+}^{\infty}\left(V_{\mathrm{coh},}^{1} E\right)^{\operatorname{Aut}(T)} \longrightarrow \ell_{+}^{\infty}\left(V_{\mathrm{coh}}^{2}, E\right)^{\operatorname{Aut}(T)} \longrightarrow \ell_{+}^{\infty}\left(V_{\mathrm{coh}}^{3}, E\right)^{\operatorname{Aut}(T)} \longrightarrow \cdots
$$

Proof. Following [2], we denote by $\ell_{\mathscr{A}}^{\infty}\left(V^{q+1}, E\right)$ the sub-space of alternating maps defined on aligned tuples. The restriction to coherent tuples thus induces an isomorphism

$$
\ell_{\mathscr{A}}^{\infty}\left(V^{q+1}, E\right) \cong \ell_{+}^{\infty}\left(V_{\text {coh }}^{q+1}, E\right) .
$$

Therefore, the first statement is simply a reformulation of Corollary 8 of [2]. Moreover, as observed there, the modules $\ell_{\mathscr{A}}^{\infty}\left(V^{q+1}, E\right)$ are relatively injective in the sense of bounded cohomology because the $\operatorname{Aut}(T)$-action on the set of aligned tuples is proper, see [9, 4.5.2]. More precisely, $\ell_{\mathscr{A}}^{\infty}\left(V^{q+1}, E\right)$ is a direct factor of the larger space without the alternation condition, to which [9, 4.5.2] applies, and one concludes as in [9, 7.4.5] by an alternation map. 
A direct computation using the relation $\operatorname{sign}\left(\tau_{q}\right) \cdot \operatorname{sign}\left(\tau_{q+1}\right)=(-1)^{q+1}$ shows that $\hat{\tau}_{q}$ is a chain map. In particular, $\hat{\tau}_{q}$ automatically preserves the decomposition $\ell_{ \pm}^{\infty}\left(V_{\mathrm{coh}}^{q+1}, E\right)$ and $A_{q}$ is a chain map as well. Now the second statement follows by general cohomological principles (see e.g. $\S 7.2$ in [9]).

\section{A PRIMITIVE FOR THE SQUARE OF $\omega$ ON COHERENT TUPLES}

We define an $\operatorname{Aut}(T)$-equivariant map

$$
B: V_{\text {coh }}^{4} \longrightarrow \mathbf{R}_{\text {alt }}[P] \otimes \mathbf{R}_{\text {alt }}[P]
$$

by setting, for any coherent 4-tuple $x$ and any paths $p_{1}, p_{2} \in P$,

$$
B(x)\left(p_{1}, p_{2}\right)= \pm 1
$$

whenever all the following hold:

- both $p_{1}$ and $p_{2}$ are carried by $\left[x_{0}, x_{3}\right]$,

- the interior of $p_{1}$ and of $p_{2}$ are disjoint,

- $x_{i} \in \operatorname{Int}\left(p_{i}\right)$ for each $i=1,2$.

In that case, the sign \pm 1 is the product of the orientations of $p_{1}$ and of $p_{2}$ relative to $\left[x_{0}, x_{3}\right]$. All this is perhaps much more intuitive in a picture, drawn for two of the four orientation possibilities:

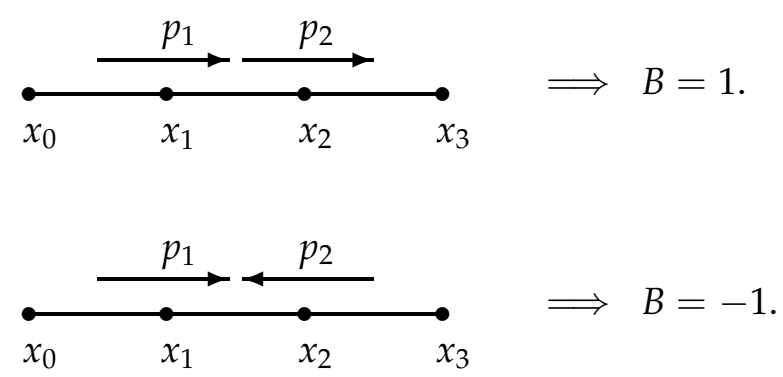

In all other cases, we set $B(x)\left(p_{1}, p_{2}\right)=0$.

Proposition 6. We have $d B(x)=\omega \smile \omega(x)$ for every coherent 5-tuple $x$.

Proof. Let $p_{1}, p_{2} \in P$. By definition,

$$
\omega \smile \omega(x)\left(p_{1}, p_{2}\right)=\omega\left(x_{0}, x_{1}, x_{2}\right)\left(p_{1}\right) \cdot \omega\left(x_{2}, x_{3}, x_{4}\right)\left(p_{2}\right) .
$$

Thus, $\omega \smile \omega(x)\left(p_{1}, p_{2}\right) \neq 0$ if and only if all the following hold:

$$
\left\{\begin{array}{l}
x_{1} \in \operatorname{Int}\left(p_{1}\right) \text { and } p_{1} \sqsubset\left[x_{0}, x_{2}\right], \\
x_{3} \in \operatorname{Int}\left(p_{2}\right) \text { and } p_{2} \sqsubset\left[x_{2}, x_{4}\right] .
\end{array}\right.
$$

As for $d B$, we observe that $d B(x)\left(p_{1}, p_{2}\right)=0$ unless possibly

$$
\left\{\begin{array}{l}
p_{1}, p_{2} \text { have disjoint interior and are carried by }\left[x_{0}, x_{4}\right] \\
x_{1} \text { or } x_{2} \in \operatorname{Int}\left(p_{1}\right) \\
x_{2} \text { or } x_{3} \in \operatorname{Int}\left(p_{2}\right) .
\end{array}\right.
$$

In the case when Conditions ( $(\mathrm{V})$ are not satisfied, Conditions (iv) are not either; therefore in that case $d B$ and $\omega \smile \omega$ agree since they both vanish. 
Suppose now that Conditions $(\mathbb{\nabla})$ are satisfied. By symmetry, we can assume that the orientation of $p_{1}$ and $p_{2}$ are compatible with the orientation of $\left[x_{0}, x_{4}\right]$ (and hence of $\left[x_{0}, x_{3}\right]$ and of $\left.\left[x_{1}, x_{4}\right]\right)$. Since $p_{1}$ and $p_{2}$ have disjoint interior, $x_{2}$ is contained in at most one of $\operatorname{Int}\left(p_{1}\right)$ or $\operatorname{Int}\left(p_{2}\right)$; we can suppose that it is not contained in $\operatorname{Int}\left(p_{1}\right)$, the other case being completely analogous. We have now three cases:

First case: $x_{1} \in \operatorname{Int}\left(p_{1}\right), x_{2} \notin \operatorname{Int}\left(p_{1}\right) \cup \operatorname{Int}\left(p_{2}\right)$ and $x_{3} \in \operatorname{Int}\left(p_{2}\right)$.

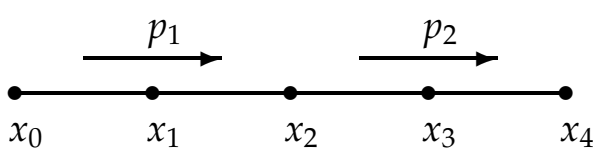

The value of $\omega \smile \omega(x)\left(p_{1}, p_{2}\right)$ is +1 , while the only non-zero summand in

$$
d B(x)\left(p_{1}, p_{2}\right)=\Sigma_{i=0}^{4}(-1)^{i} B\left(\ldots, \widehat{x}_{i}, \ldots\right)
$$

is the one for $i=2$, which is indeed also +1 .

Second case: $x_{1} \in \operatorname{Int}\left(p_{1}\right)$ and $x_{2}, x_{3} \in \operatorname{Int}\left(p_{2}\right)$.

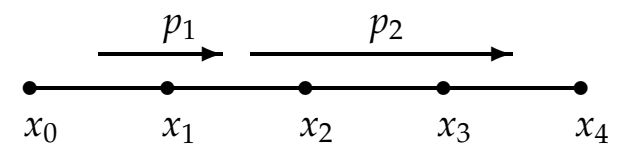

Condition (iv) is not satisfied and hence $\omega \smile \omega$ vanishes. As for $d B$, only the summands for $i=2$ and $i=3$ are non-zero and cancel out to give $d B(x)\left(p_{1}, p_{2}\right)=0$.

Third case: $x_{1} \in \operatorname{Int}\left(p_{1}\right), x_{2} \in \operatorname{Int}\left(p_{2}\right)$ and $x_{3} \notin \operatorname{Int}\left(p_{2}\right)$.

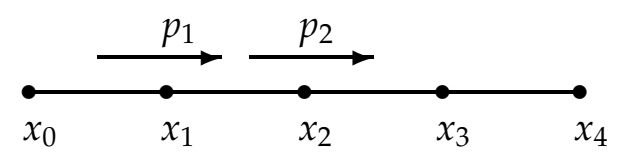

Again, condition $(\bar{\nabla})$ is not satisfied and $\omega \smile \omega$ vanishes. As for $d B$, only the summands for $i=3$ and $i=4$ are non-zero and cancel out to give $d B(x)\left(p_{1}, p_{2}\right)=0$.

\section{Proof of Theorem 4}

We first verify that the primitive $B$ is bounded.

Lemma 7. The map B is uniformly bounded on $V_{\mathrm{coh}}^{4}$ with respect to the projective norm $\|\cdot\|_{\pi}$.

Proof. Fix $x \in V_{\text {coh }}^{4}$ and consider abusively any path $p_{i}$ as an element of $\mathbf{R}[P]$. By definition of the projective cross-norm, we can bound $\|B(x)\|_{\pi}$ by $\sum\left(\left\|p_{1}\right\|_{\text {path }} \cdot\left\|p_{2}\right\|_{\text {path }}\right)$, where the sum runs over all pairs $\left(p_{1}, p_{2}\right)$ on which $B(x)$ does not vanish. Arguing as in our estimate for the norm of $\omega$, we have at most $2\left(n_{1}-1\right)\left(n_{2}-1\right)$ such pairs whenever we fix the length $n_{i}$ of 
each $p_{i}$. Since on the other hand we have $\left\|p_{i}\right\|_{\text {path }}=1 / n_{i} !$, we conclude that $B(x)$ has norm at most

$$
\sum_{n_{1}, n_{2}} \frac{2\left(n_{1}-1\right)\left(n_{2}-1\right)}{n_{1} ! n_{2} !}=2\left(\sum_{n} \frac{n-1}{n !}\right)^{2}=2 .
$$

At this point, we conclude that $A_{3}(B)$ belongs to $\ell_{+}^{\infty}\left(V_{\text {coh' }}^{4}, \mathscr{P}\right)$. Since $A_{*}$ is a chain map (as pointed out in the proof of Proposition [5), we deduce from Proposition 6 that we have $A_{4}(\omega \smile \omega)=d A_{3}(B)$. Now Proposition 5 implies that the class of $\omega \smile \omega$ vanishes, completing the proof of Theorem 4 .

Acknowledgements. The authors are grateful to Tobias Hartnick for his comments.

\section{REFERENCES}

[1] Robert Brooks, Some remarks on bounded cohomology, Riemann surfaces and related topics: Proceedings of the 1978 Stony Brook Conference (State Univ. New York, Stony Brook, N.Y., 1978), Ann. of Math. Stud., vol. 97, Princeton Univ. Press, 1981, pp. 53-63.

[2] Michelle Bucher and Nicolas Monod, The bounded cohomology of SL2 over local fields and S-integers, 2017, IMRN, in press.

[3] Bruno Duchesne and Nicolas Monod, Group actions on dendrites and curves, Preprint, arxiv:1609.00303v2, 2016.

[4] Rostislav I. Grigorchuk, Some results on bounded cohomology, Combinatorial and geometric group theory (Edinburgh, 1993), London Math. Soc. Lecture Note Ser., vol. 204, Cambridge Univ. Press, Cambridge, 1995, pp. 111-163.

[5] Tobias Hartnick and Pascal Schweitzer, On quasioutomorphism groups of free groups and their transitivity properties, J. Algebra 450 (2016), 242-281.

[6] Nicolaus Heuer, Cup product in bounded cohomology of the free group, Preprint, arxiv:1710.03193, 2017.

[7] Barry E. Johnson, Cohomology in Banach algebras, AMS, 1972, Mem. Am. Math. Soc. 127.

[8] Yoshihiko Mitsumatsu, Bounded cohomology and $l^{1}$-homology of surfaces, Topology 23 (1984), no. 4, 465-471.

[9] Nicolas Monod, Continuous bounded cohomology of locally compact groups, Lecture Notes in Mathematics 1758, Springer, Berlin, 2001.

[10] Nicolas Monod and Yehuda Shalom, Negative curvature from a cohomological viewpoint and cocycle superrigidity, C. R. Acad. Sci. Paris Sér. I Math. 337 (2003), no. 10, 635-638.

[11] _ Cocycle superrigidity and bounded cohomology for negatively curved spaces, Journal of Differential Geometry 67 (2004), 395-455.

[12] Akbar Hussein Rhemtulla, A problem of bounded expressibility in free products, Proc. Cambridge Philos. Soc. 64 (1968), 573-584.

[13] Raymond A. Ryan, Introduction to tensor products of Banach spaces, Springer Monographs in Mathematics, Springer-Verlag London, Ltd., London, 2002.

University of GENEVA, 1211 GENEVA 4, SWITZERLAND

E-mail address: michelle.bucher-karlsson@unige.ch

EPFL, SB-MATH-EGG, 1015 LAUSANNE, SWITZERLAND

E-mail address: nicolas.monod@epf $1 . \mathrm{ch}$ 